\title{
Review on Rock Aquifers Researches Conducted in Malaysia
}

\author{
Vynotdni Rathinasamy ${ }^{1}$, Edy Tonnizam Mohammad ${ }^{2 *}$, and Ibrahim Komoo ${ }^{3}$ \\ ${ }^{1}$ Department of Geotechnics and Transportation, 811300 Skudai, Johor \\ ${ }^{2}$ Centre of Tropical Geoengineering (Geotropik), 811300 Skudai, Johor \\ ${ }^{3}$ Institute for Environment and Development (LESTARI), 43600 UKM Bangi, Selangor
}

\begin{abstract}
The exploration of groundwater in Malaysia was not very welcomed due to few misconceptions such as it is very expensive to extract, could give negative impacts to the environment and not reliable. Yet, in recent times, rock aquifers such as limestone, sedimentary rocks, volcanic rocks, igneous rocks and metasedimetary rocks are being explored. This paper aims to review the researches that have been carried out on rock aquifers in Malaysia. In general, the topics studied were potential zonation, groundwater quality and quantity, occurrence and flow of groundwater as well as characterization of rock aquifers. The highest yielding aquifer was metasedimentary rock aquifers meanwhile the quality was good in general. Most of the aquifers were having neutral $\mathrm{pH}$ values and the total dissolved solids more than $100 \mathrm{mg} / \mathrm{l}$. Moreover, granitic aquifers contained more hardness than the metasedimentary rocks. The iron content in the aquifers was higher than the limit set by World Health Organisation which is $0.3 \mathrm{mg} / \mathrm{l}$ while salinity of aquifers were not tested in many aquifers. It can be vividly seen that many research were focused on quality. Hence, more researches on characterization of rock aquifers must be done for proper understanding on occurrence, flow and recharge.
\end{abstract}

\section{Introduction}

The main water resource that intensely being used in Malaysia is the surface water eventhough the country is rich with other water resources like groundwater. The groundwater extraction is not embraced in here due to few misconceptions. The misconceptions are groundwater is limited, contaminated, not reliable, difficult to extract, expensive to drill and could cause harm to environment [1]. The other factors preventing exploration of groundwater are budget contrain and insufficient strategic plan for groundwater development. However, in the year 2016, the government has decided to integrate aquifer systems in water supply system up to $30 \%$ by the year 2030 [2]. It is considered a huge milestone in Malaysian water resources management. Recently, more rock aquifers are being explored in Malaysia.

\footnotetext{
*Corresponding author: edy@utm.my
} 


\section{Literature review}

Rock aquifers are explored to a certain extent in almost all the states in Malaysia. The common types of rock aquifers are limestone, metasedimentary rock aquifers, sedimentary rock aquifers, volcanic aquifers and igneous rock aquifers [1, 3, 4]. First, the metasedimentary rock aquifers, sedimentary rock with volcanic tuff aquifers and granitic aquifers were found in Negeri Sembilan [5]. The purpose of the study was to carry out fracture analysis and around 71 wells were drilled. Metasedimentary rock aquifers were drilled to a maximum depth of $187 \mathrm{~m}$ with an average depth of $125 \mathrm{~m}$ which produced a maximum yield of $960 \mathrm{~m} 3 /$ day. As for the quality, Total Dissolved Solid (TDS) was $111 \mathrm{mg} / \mathrm{L}$, hardness was from 52 to $97 \mathrm{mg} / \mathrm{L}$ and iron content $(\mathrm{Fe})$ was 5.09 . Next, sedimentary rock with volcanc tuff aquifers were drilled to a maximum depth of $200 \mathrm{~m}$ with an average depth of $163 \mathrm{~m}$ which produced a maximum yield of $1264 \mathrm{~m} 3 / \mathrm{day}$. On the other hand, Granitic aquifers were drilled to a maximum depth of $138 \mathrm{~m}$ with an average depth of $100 \mathrm{~m}$ which produced a maximum yield of $650 \mathrm{~m} 3 /$ day and average yield of $400 \mathrm{~m} 3 /$ day. As for the quality, $\mathrm{pH}$ value was 7.2 , TDS was $170 \mathrm{mg} / \mathrm{L}$, hardness was from 43 to $152 \mathrm{mg} / \mathrm{L}$ and Fe was 0.98 . The end results of this study were fracture orientations were linked to rivers and the fracture pattern followed the regional structural trend of the Main Range Granite.

The characteristics of groundwater in granitic and metasedimentary fractured rocks at West Coast Malaysia were determined [6]. Around 133 wells with depth between 100 and $200 \mathrm{~m}$ were studied. The Metasedimentary rock aquifers produced an average of $400 \mathrm{~m} 3 /$ day while Granitic aquifers produced an average of $300 \mathrm{~m} 3 /$ day. The $\mathrm{pH}$ was 6.5 , TDS was $120 \mathrm{mg} / \mathrm{L}$ and $\mathrm{Fe}$ was $1.5 \mathrm{mg} / \mathrm{L}$ when tested for quality. Furthermore, groundwater quality of interbedded sandstone, shale and musdstone at Shah Alam was studied [7]. The groundwater samples were collectected from 31 wells which were more $100 \mathrm{~m}$ in depth and tested for quality. It was found that the geology of study area controlled the catchement area and quality of groundwater was good. Yet, it was forecasted that the aquifer could contaminate if pollution occurred locally. Next, the research on quantity and quality of metasedimentary rock aquifers around Melaka, Negeri Sembilan, Selangor and Kedah was carried out [8]. Around 58 wells were studied with maximum depth of $201 \mathrm{~m}$ and average depth of $146 \mathrm{~m}$. The maximum yield was $890 \mathrm{~m} 3 /$ day while the average yield was $381 \mathrm{~m} 3 /$ day. In addition, the samples collected had TDS from 100 to $150 \mathrm{mg} / \mathrm{L}$, hardness lesser than $60 \mathrm{mg} / \mathrm{L}$ and $\mathrm{Fe}$ of 2.39 .

Moreover, mapping of potential aquifer in Selangor and Negeri Sembilan using remote sensing and GIS technique was done [9]. Geology of the study area was granite, sedimentary rocks with volcanic intercalation and metasedimentary rocks. When validated the map using wells, the results showed an accuracy of $84.74 \%$. In addition, the wells produced more than $264 \mathrm{~m} 3 /$ day. Later, the study on potential aquifer zonation in Kedah and Perlis was carried out [10]. The lithology of Kedah was Chert while the lithology of Perlis was and crystalline limestone, mudstone and semi-consolidated gravel. Around 16 wells were drilled to validate the prediction and it was accurate up to $81.25 \%$. The maximum yield was $632 \mathrm{~m} 3 /$ day while average was $230.88 \mathrm{~m} 3 /$ day. Next, the factors influencing groundwater chemistry was studied at Kapas Island, Terengaanu [11]. Around 18 wells were drilled for this purpose. The samples showed $\mathrm{pH}$ value was 6.9, TDS was 988.3 and salinity (Sal) was 0.9 when tested. It was found that seawater intrusion, weathering, redox reaction and anthropogenic pollution greatly effect the quality of aquifers in this island.

A study on hydraulic and contamination stress in metasedimentary rock aquifer at Shah Alam was conducted as well [12]. It result showed that the aquifer was prone quality stress as only limited contamination occurred in the study area. However, the pumping can effect the quality stress further. In addition, groundwater quality study was carried out in Kajang 


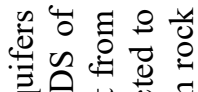

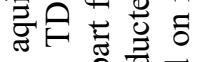

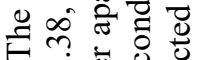

.

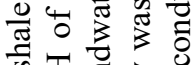

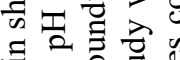

记

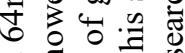

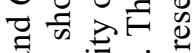

क

唡

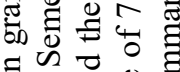

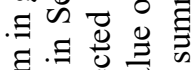

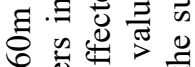

0.00

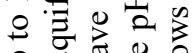

?.

क क क क

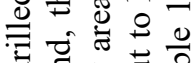

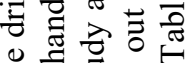

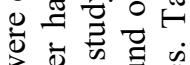

उ) क

曲

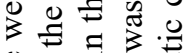

들

EO

๓

च

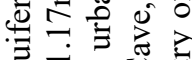

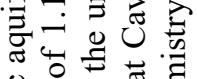

. 00 苞

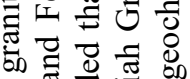

흘 긍

드엘

丸ઢ

ข점크

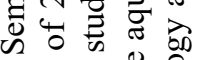

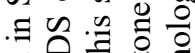

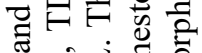
ஸี กิ่

范 导

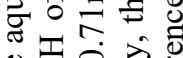

늘

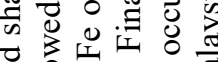

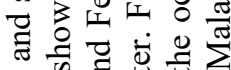

.气

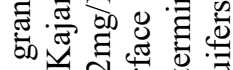

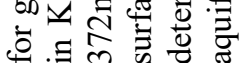

\begin{tabular}{|c|c|c|c|c|c|c|c|}
\hline \multirow{10}{*}{ 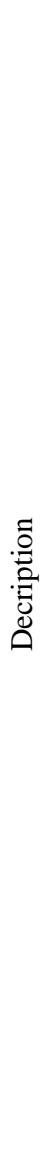 } & \multirow{5}{*}{ 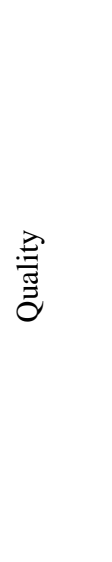 } & 胥 & ' & ' & ' & \multicolumn{2}{|l|}{ ' } \\
\hline & & II & $\begin{array}{l}\dot{\partial} \\
\dot{n}\end{array}$ & ' & $\stackrel{\infty}{\stackrel{0}{0}}$ & \multicolumn{2}{|l|}{$\stackrel{n}{-}$} \\
\hline & & 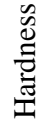 & î̀ & ' & $\frac{n}{\frac{n}{2}}$ & \multicolumn{2}{|l|}{ ' } \\
\hline & & $\tilde{\theta}$ & $\Xi$ & ' & $\stackrel{尺}{ }$ & \multicolumn{2}{|l|}{ 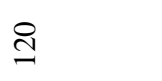 } \\
\hline & & I & ' & ' & $\stackrel{N}{r}$ & \multicolumn{2}{|l|}{$\tilde{\sigma}$} \\
\hline & \multirow{2}{*}{ 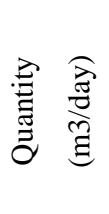 } & $\stackrel{\infty}{\gtrless}$ & ' & ' & \& & \& & ষ্ల \\
\hline & & 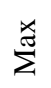 & \& & $\begin{array}{l}\mathbb{U} \\
\text { త }\end{array}$ & ஜุ & \multicolumn{2}{|l|}{$\underset{\infty}{\varnothing}$} \\
\hline & \multirow{2}{*}{ 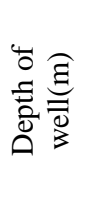 } & $\stackrel{\infty}{<}^{\infty}$ & 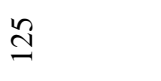 & $\hat{\sigma}$ & 8 & \multicolumn{2}{|l|}{ \&) } \\
\hline & & 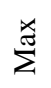 & $\stackrel{\infty}{-\infty}$ & ஓి & $\stackrel{\infty}{=}$ & ' & \\
\hline & \multicolumn{2}{|l|}{$\begin{array}{l}\text { to } \\
\dot{z} \\
\dot{z}\end{array}$} & \multicolumn{3}{|l|}{$\nabla$} & \multicolumn{2}{|l|}{$\stackrel{m}{n}$} \\
\hline \multicolumn{2}{|c|}{ 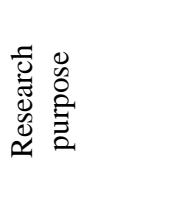 } & & \multicolumn{3}{|l|}{ 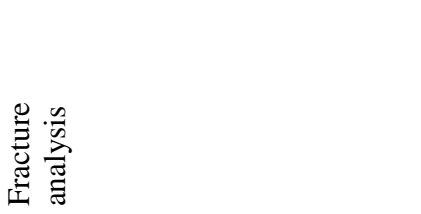 } & \multicolumn{2}{|l|}{ 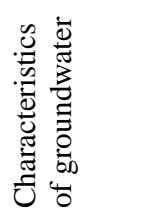 } \\
\hline \multicolumn{3}{|c|}{ 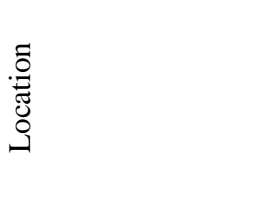 } & \multicolumn{3}{|l|}{ 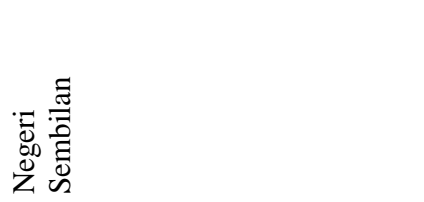 } & \multicolumn{2}{|c|}{ 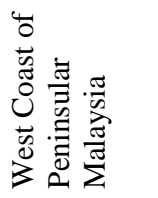 } \\
\hline \multicolumn{3}{|c|}{$\begin{array}{l}\text { के } \\
\frac{0}{0} \\
\stackrel{0}{0}\end{array}$} & 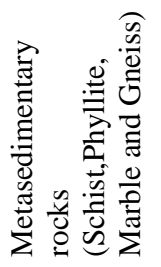 & 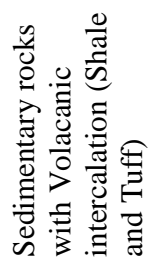 & 莺 & 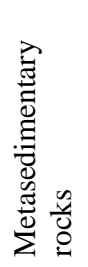 & 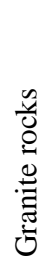 \\
\hline \multicolumn{3}{|c|}{$\begin{array}{l}\ddot{0} \\
\ddot{\Xi} \\
\ddot{0}\end{array}$} & \multicolumn{3}{|l|}{$\sqrt{n}$} & \multicolumn{2}{|l|}{$\sigma$} \\
\hline
\end{tabular}




\begin{tabular}{|c|c|c|c|c|c|c|}
\hline . & , & \multicolumn{3}{|l|}{ ' } & , & , \\
\hline ' & ले & \multicolumn{3}{|l|}{ ' } & \multicolumn{2}{|l|}{ ' } \\
\hline , & $\underset{8}{8}$ & \multicolumn{3}{|l|}{, } & \multicolumn{2}{|l|}{ ' } \\
\hline$\frac{\&}{v}$ & 8 & \multicolumn{3}{|l|}{ ' } & \multicolumn{2}{|l|}{ ' } \\
\hline 它怘 & $\bar{\infty}$ & \multicolumn{3}{|l|}{ 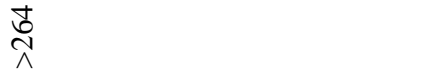 } & \multicolumn{2}{|l|}{$\overline{\vec{\lambda}}$} \\
\hline 8 & ஓి & \multicolumn{3}{|l|}{ ' } & \multicolumn{2}{|l|}{ గ్రి } \\
\hline$\underset{\lambda}{\stackrel{8}{\circ}}$ & I & \multicolumn{3}{|l|}{ ' } & \multicolumn{2}{|l|}{ ' } \\
\hline ' & $\overrightarrow{\stackrel{\nu}{~}}$ & \multicolumn{3}{|l|}{ ' } & \multicolumn{2}{|l|}{ ' } \\
\hline $\bar{m}$ & $\stackrel{\infty}{n}$ & \multicolumn{3}{|l|}{ 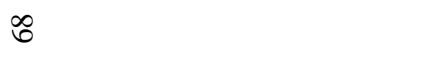 } & \multicolumn{2}{|l|}{$\stackrel{0}{0}$} \\
\hline 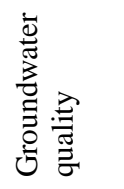 & 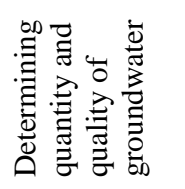 & \multicolumn{3}{|l|}{ 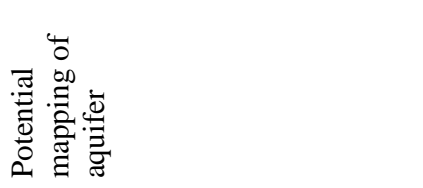 } & \multicolumn{2}{|c|}{ 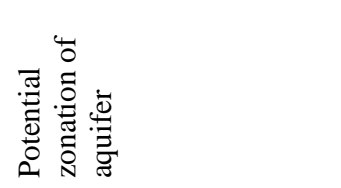 } \\
\hline 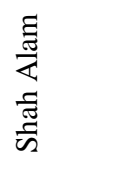 & 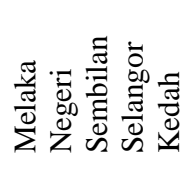 & \multicolumn{3}{|l|}{ 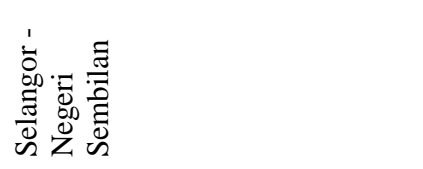 } & 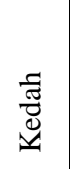 & 堷 \\
\hline 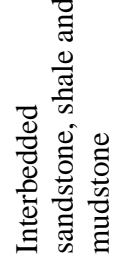 & 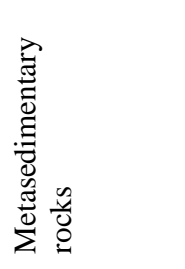 & 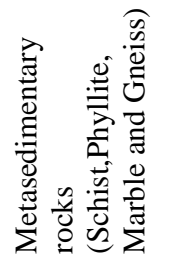 & 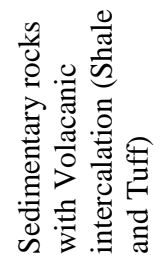 & 穿 & $\begin{array}{l}\text { 离 } \\
\text { U }\end{array}$ & 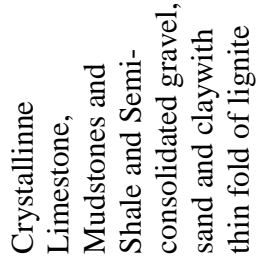 \\
\hline$\Sigma$ & $\underline{\infty}$ & $\bar{\Omega}$ & & & $\stackrel{\varrho}{\varrho}$ & \\
\hline
\end{tabular}




\begin{tabular}{|c|c|c|c|c|c|}
\hline \multicolumn{2}{|c|}{$\dot{0}$} & ' & \multicolumn{3}{|l|}{ ' } \\
\hline \multicolumn{2}{|l|}{ ' } & $\stackrel{+}{i}$ & \multicolumn{2}{|l|}{$\cong$} & $\stackrel{\vec{t}}{\circ}$ \\
\hline \multicolumn{2}{|l|}{ ' } & , & \multicolumn{3}{|l|}{ ' } \\
\hline \multicolumn{2}{|l|}{$\begin{array}{l}\infty \\
\infty \\
\infty\end{array}$} & $\underset{\infty}{\infty}$ & \multicolumn{2}{|l|}{ 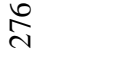 } & 离 \\
\hline \multicolumn{2}{|l|}{ ڤे) } & 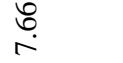 & \multicolumn{2}{|l|}{$\stackrel{n}{r}$} & $\begin{array}{c}\infty \\
\tilde{\sigma}^{2}\end{array}$ \\
\hline \multicolumn{2}{|l|}{, } & ' & \multicolumn{3}{|l|}{ ' } \\
\hline \multicolumn{2}{|l|}{, } & in & \multicolumn{3}{|l|}{ ' } \\
\hline \multicolumn{2}{|l|}{, } & ' & $\stackrel{\beth}{\beth}$ & ' & $\tilde{I}$ \\
\hline \multicolumn{2}{|l|}{ ' } & 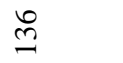 & $\stackrel{8}{0}$ & t & $\stackrel{8}{0}$ \\
\hline \multicolumn{2}{|l|}{$\stackrel{\infty}{=}$} & 㛐 & $\sim$ & - & $N$ \\
\hline \multicolumn{2}{|l|}{ 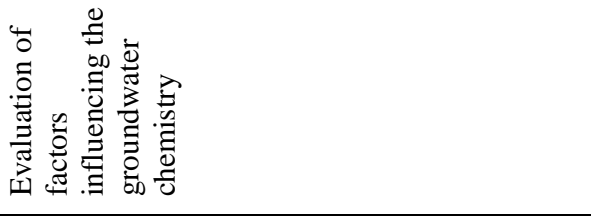 } & 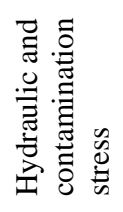 & \multicolumn{3}{|c|}{ 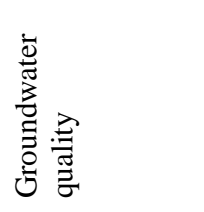 } \\
\hline \multicolumn{2}{|l|}{ 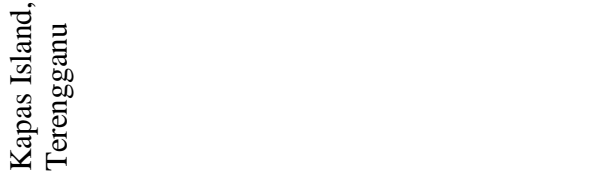 } & 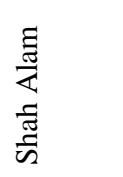 & \multicolumn{2}{|l|}{ 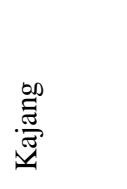 } & 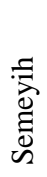 \\
\hline 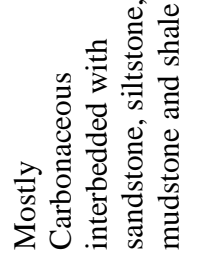 & 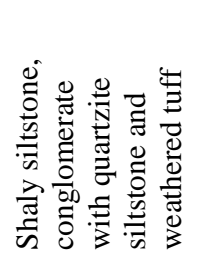 & 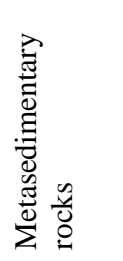 & 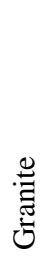 & $\frac{0}{\frac{\pi}{\omega n}}$ & 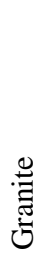 \\
\hline \multicolumn{2}{|l|}{$\Xi$} & $\bar{\Xi}$ & \multicolumn{3}{|l|}{$\stackrel{\bar{\Xi}}{\Xi}$} \\
\hline
\end{tabular}




\begin{tabular}{|c|}
\hline , \\
\hline , \\
\hline , \\
\hline , \\
\hline$r$ \\
\hline ' \\
\hline ' \\
\hline ' \\
\hline ' \\
\hline , \\
\hline 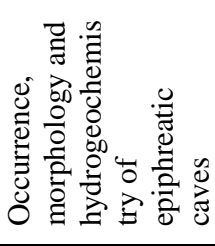 \\
\hline 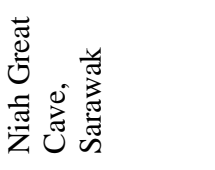 \\
\hline 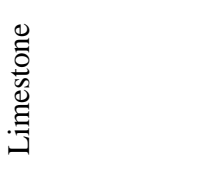 \\
\hline$\Xi$ \\
\hline
\end{tabular}

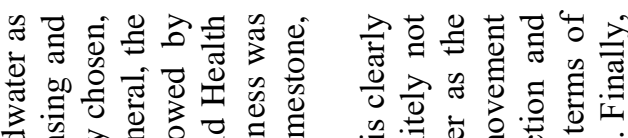

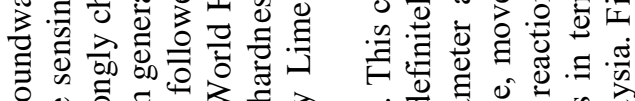

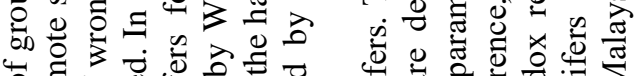
ডั

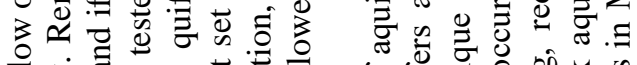
柁灾

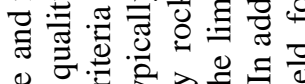

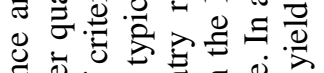

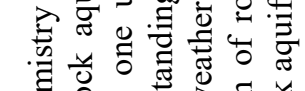

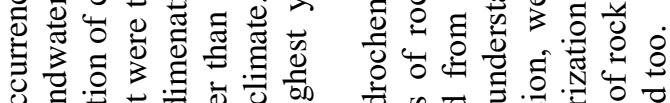

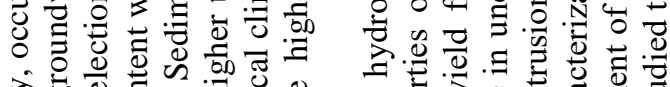

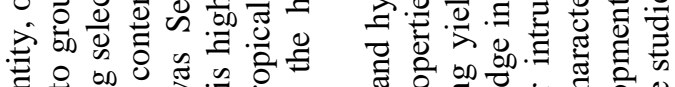

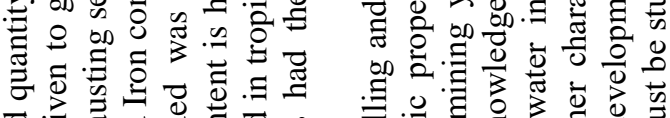

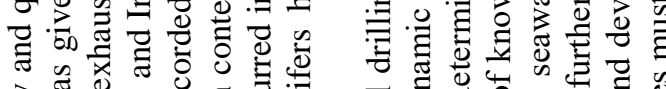

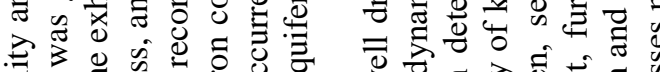

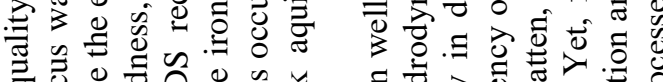

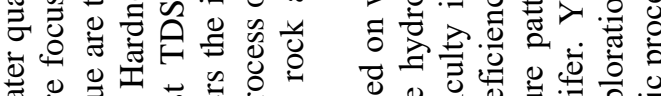

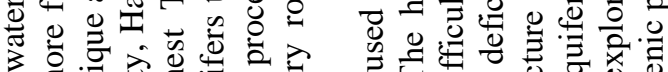

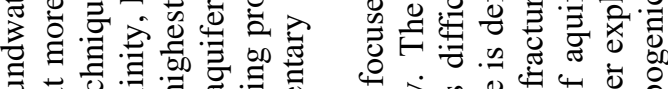

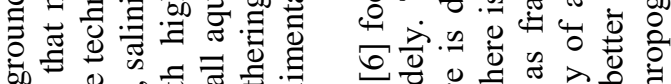

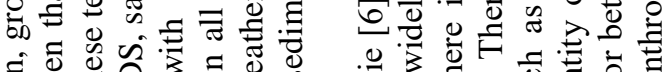

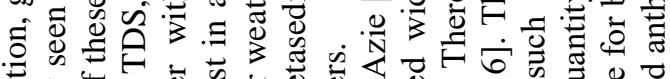

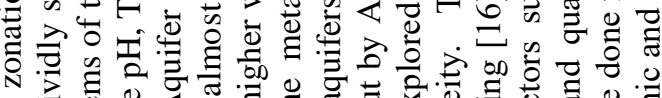

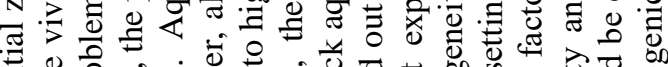

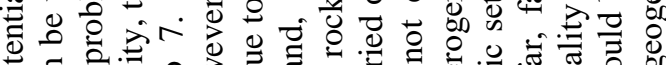

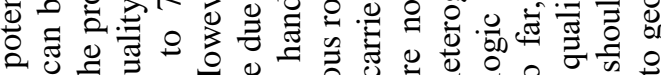

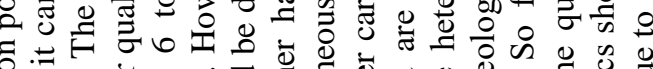

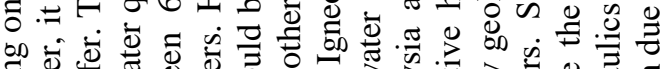

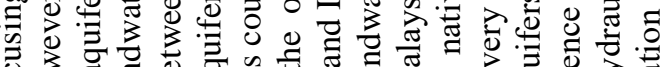

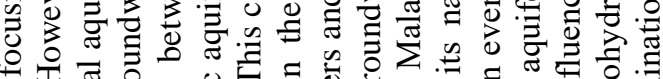

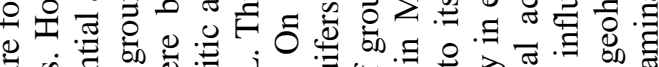
은

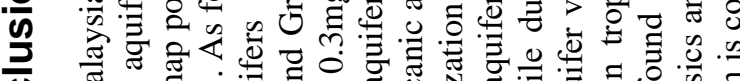

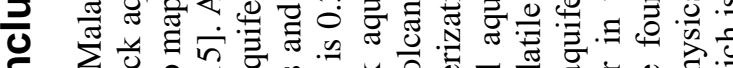

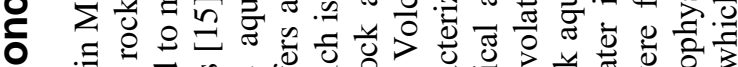

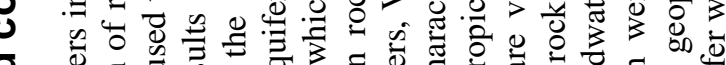
능 त

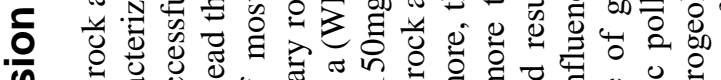
员

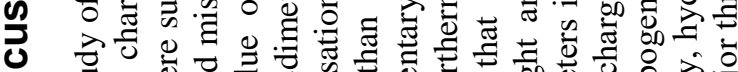

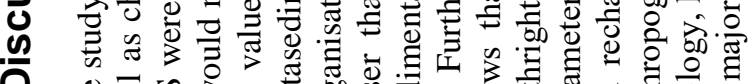
m 
The authors would like to express their appreciation for the scholarship support of Zamalah Scholarship of Universiti Teknologi Malaysia.

\section{References}

1. Y.A. Razak, M.H.A. Karim. Groundwater in the Malaysian Context. Groundwater Management in Malaysia-Status and Challenges. Putrajaya. Minerals and Geoscience Department Malaysia \& Academy of Sciences Malaysia. 1-14. (2009)

2. F. Chand. Integrated Aquifer System Management Journal. (Issue). 15-31. (2016)

3. Z. Yahya, S. Suratman. Hard Rock Aquifers in Peninsular Malaysia Journal. (Issue). 102-118. (2009)

4. F.S. Chong, D.N.K. Tan. Hydrogeological activities in Peninsular Malaysia and Sarawak. GEOSEA V Proceedings. Malaysia. Bulletin Of The Geological Society Of Malaysia. 827-842. (1986)

5. Nasiman, R. Zainariah, F. Ramli. Fracture pattern and its relationship to groundwater in hardrocks of Negeri Sembilan Annual Geological Conference '96. Kota Kinabalu. Bulletin Of The Geological Society Of Malaysia. 113-118. (1996)

6. R.Z.B.R. Azie. Characteristics Of Groundwater From Fractured Hardrocks In West Coast Of Peninsular Malaysia. Pages. (1997)

7. A. Zuber, J. Kania, E. Kmiecik. The impact of recent urbanization on a hard rock aquifer in Malaysia. XXXVIII IAH Congress. Poland. (2010)

8. N. Sapari, R.Z.R. Azie, H. Jusoh. Quantity and Quality of Groundwater in Fractured Metasedimentary Rocks of the West Coast of Peninsular Malaysia Sains Malaysiana. 40, (6). 537-542 (2011)

9. M.A. Manap, H. Nampak, B. Pradhan, S. Lee, W.N.A. Sulaiman, M.F. Ramli. Application of probabilistic-based frequency ratio model in groundwater potential mapping using remote sensing data and GIS. Arabian Journal of Geosciences. 7, (2). 711-724. (2012)

10. K.A.N. Adiat, M.N.M. Nawawi, K. Abdullah. Assessing the accuracy of GISbased elementary multi criteria decision analysis as a spatial prediction tool - A case of predicting potential zones of sustainable groundwater resources. Journal of Hydrology. 440-441. 75-89. (2012)

11. N.U. Kura, M.F. Ramli, W.N. Sulaiman, S. Ibrahim, A.Z. Aris, A. Mustapha. Evaluation of factors influencing the groundwater chemistry in a small tropical island of Malaysia. Int J Environ Res Public Health. 10, (5). 1861-81. (2013)

12. N. Roslan. The Potential Susceptibility Of Urban Hardrock Aquifers To Hydraulic and Contaminant Stress: The case of Shah Alam, Malaysia. Pages. (2017)

13. S. Haque, N. Roslan. Groundwater Quality Aspect Owing to Urbanization in Langat Basin (Kajang, Semenyih) Malaysia. Asian Journal of Environment \& Ecology. 4, (2). 1-9. (2017)

14. D. Dodge-Wan, M.V. Prasanna, R. Nagarajan, A. Anandkumar. Epiphreatic Caves In Niah Karst Tower (NW Borneo): Occurrence, Morphology And Hydrogeochemistry. ACTA CARSOLOGICA 46, (2-3). 149-153. (2017)

15. H. Yin, Y. Shi, H. Niu, D. Xie, J. Wei, L. Lefticariu, S. Xu. A GIS-based model of potential groundwater yield zonation for a sandstone aquifer in the Juye Coalfield, Shangdong, China. Journal of Hydrology. 557. 434-447. (2018)

16. M. Holland. Hydrogeological characterisation of crystalline basement aquifers within the Limpopo Province, South Africa Pages. (2011) 